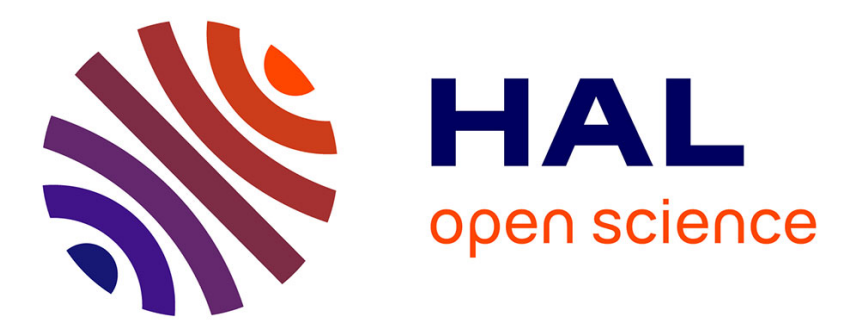

\title{
On-Wafer Broadband Microwave Measurement of High impedance Devices-CPW Test Structures with Integrated Metallic Nano-resistances
}

K. Daffe, F. Mubarak, V. Mascolo, H. Votsi, N. Ridler, Gilles Dambrine, Isabelle Roch-Jeune, Kamel Haddadi

\section{To cite this version:}

K. Daffe, F. Mubarak, V. Mascolo, H. Votsi, N. Ridler, et al.. On-Wafer Broadband Microwave Measurement of High impedance Devices-CPW Test Structures with Integrated Metallic Nanoresistances. 2018 48th European Microwave Conference (EuMC), Sep 2018, Madrid, Spain. pp.25-28, 10.23919/EuMC.2018.8541607 . hal-02056825

\section{HAL Id: hal-02056825 \\ https://hal.science/hal-02056825}

Submitted on 4 Mar 2019

HAL is a multi-disciplinary open access archive for the deposit and dissemination of scientific research documents, whether they are published or not. The documents may come from teaching and research institutions in France or abroad, or from public or private research centers.
L'archive ouverte pluridisciplinaire HAL, est destinée au dépôt et à la diffusion de documents scientifiques de niveau recherche, publiés ou non, émanant des établissements d'enseignement et de recherche français ou étrangers, des laboratoires publics ou privés. 


\title{
On-Wafer Broadband Microwave Measurement of High impedance Devices - CPW Test Structures with Integrated Metallic Nano-resistances
}

\author{
K. Daffe ${ }^{\#}$, F. Mubarak ${ }^{* 2}$, V. Mascolo*, G. Dambrine ${ }^{\#}$, I. Roch ${ }^{\#}$ and K. Haddadi ${ }^{\# 1}$ \\ "University Lille, CNRS, UMR 8520 - IEMN, F-59000 Lille, France \\ *VSL, Van Swinden Laboratorium, Delft, The Netherlands \\ ${ }^{1}$ kamel.haddadi@univ-lille1.fr, ${ }^{2}$ fmubarak@vsl.nl
}

\begin{abstract}
On-wafer microwave characterization and uncertainty evaluation of two-port coplanar waveguide (CPW) high impedance nanodevices devices are proposed. The test vehicles are built up with resistive metallic nano-films integrated in tapered CPW structures. Microwave conductance in the range $100-500 \mu \mathrm{S}$ associated to parallel capacitances in the order of hundreds aF are exemplary shown up to $20 \mathrm{GHz}$. In addition, the uncertainty related to the post-calibration residual errors terms together with a sensitivity study to the technological process variability using FEM-based EM modelling are considered.
\end{abstract}

Keywords - On-wafer calibration, vector network analyzer (VNA), two-port measurement, coplanar waveguide (CPW), uncertainty.

\section{INTRODUCTION}

The microwave characterization of extremely high impedance devices is still challenging [1]. The issue of dealing with extreme impedance measurement has yielded to a variety of measurement techniques involving nanodevices embedded in CPW test structures. In 2005, the first measurements of the high frequency conductance of a metallic single walled nanotube (SWNT) with resistance below $200 \mathrm{k} \Omega$ inserted in a $\mathrm{CPW}$ transmission line were performed up to $10 \mathrm{GHz}$ [2]. In 2008, an on-wafer technique and calibration method are developed for broadband electrical characterization of $\mathrm{GaN}$ nanowires up to $40 \mathrm{GHz}$ [3]. Recently, [4]-[5] reported progress towards establishing traceability for a planar environment and reported detailed uncertainty budgets.

In this work, a conventional device characterization set-up, i.e. a vector network analyzer (VNA) connected to a pair of GSG probes, is proposed in a CPW series-through measurement configuration to tackle the issue of high impedance microwave measurement. The test vehicles chosen consist of nano-resistances ranging from 2 to $10 \mathrm{k} \Omega$ embedded in CPW test structures. Section II described the technological fabrication of the nanodevices. Microwave measurements including calibration and de-embedding methods developed in Section II demonstrate accurate microwave conductance extraction up to $20 \mathrm{GHz}$. The impact of the post-calibration residual errors terms and the technological process variability using FEM-based EM modelling for low RF conductance and capacitance values determination are presented in Section IV.

\section{TECHNOLOGY AND FABRICATION}

The high impedance devices are fabricated by integrating nano-resistances within gaps of CPW test structures with ended tapered transmission lines (Fig. 1). The fabrication process on a 3 inches semi-insulating Gallium Arsenide (GaAs) wafer, $625 \mu \mathrm{m}(+/-25 \mu \mathrm{m})$ thickness, was divided in two main steps. First step was the definition of nano-resistances $(100 \mathrm{~nm}$ width). A resist bilayer was spin-coated and e-beam lithographed with a nanomasker EBPG5000plus (Vistec ${ }^{\mathrm{TM}}$ ). Then, a $31 \mathrm{~nm}$ titanium (Ti) layer (measured mean value) was evaporated and the lift-off was achieved to form a set of nanoresistances (48.8 $\Omega$ sheet resistance), which theoretical resistance values vary from 2 to $10 \mathrm{k} \Omega$. Second step was the definition of CPW access pads. The spin-coating, the alignment and e-beam writing of a new resist bilayer were performed. Afterwards, a $25 \mathrm{~nm}$ adhesion Ti layer and a $500 \mathrm{~nm}$ gold $\mathrm{(Au})$ layer were deposited and lifted-off. For instance, Fig. 1 shows a $4 \mu \mathrm{m}$ gap test structure with integrated Ti nano-resistance.

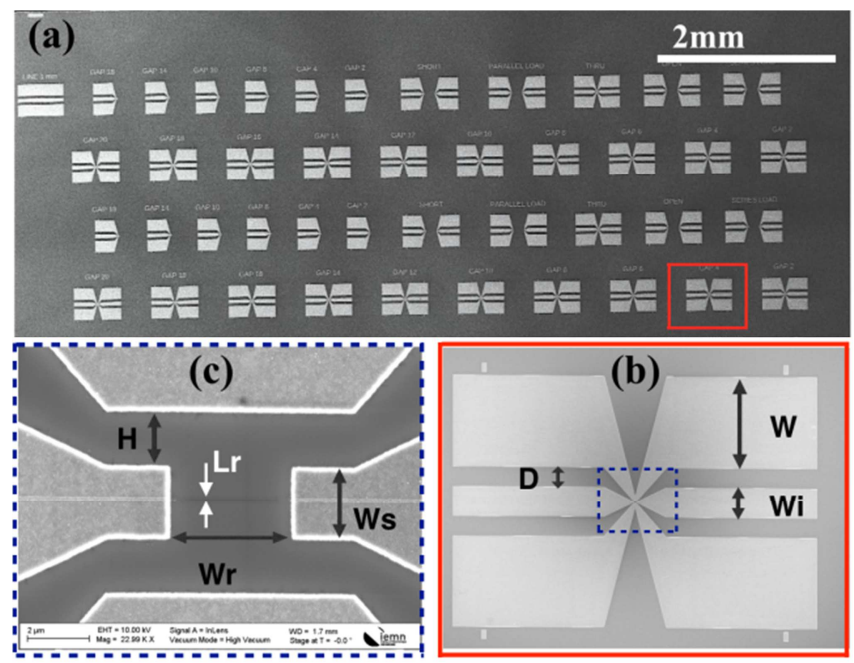

Fig.1 Scanning electron microscopy (SEM) images of (a) the general view of the wafer, (b) the zoomed view of $4 \mu \mathrm{m}$ gap with the CPW accesses and (c) magnified view of the $4 \mu \mathrm{m}$ gap with integrated nano-resistance. 
After fabrication, a Tescan ${ }^{\mathrm{TM}}$ Mira XMU SEM is used for the measurement of the critical dimensional parameters. For the Ti thickness, a white-light interferometer is used. The sheet resistance is measured using a standard four-point measurement technique. In TABLE I, both nominal and measured parameters are given for a $4 \mu \mathrm{m}$ gap device and for a $20 \mu \mathrm{m}$ empty gap device (used for de-embedding procedure).

TABLE I

PARAMETER MEASUREMENT VALUES FOR CPW DEVICES

\begin{tabular}{|c|c|c|}
\hline Parameters & $\begin{array}{c}4 \mu m \text { gap } \\
\text { (DUT) } \\
\text { Meas. [nom] }\end{array}$ & $\begin{array}{c}20 \mu \mathrm{m} \text { gap } \\
\text { (de-embed.) } \\
\text { Meas. [nom] }\end{array}$ \\
\hline$W i(\mu m)$ & 102 [100] & 102 [100] \\
\hline$W(\mu m)$ & 299 [300] & 299 [300] \\
\hline$D(\mu m)$ & $65[66]$ & $65[66]$ \\
\hline$H(\mu m)$ & $1.7[1.8]$ & $1.7[1.8]$ \\
\hline$W s(\mu m)$ & 2.4 [2.3] & 2.4 [2.3] \\
\hline$W r(\mu m)$ & $3.9[4]$ & 19 [20] \\
\hline $\operatorname{Lr}(\mathrm{nm})$ & 110 [100] & 115 [100] \\
\hline
\end{tabular}

\section{EXPERIMENTAL SET-UP AND MEASUREMENTS}

\section{A. Experimental set-up and vector calibration.}

The experimental setup involves a MPS150 conventional on-wafer probe station from Cascade Microtech ${ }^{\circledR}$ which equipped with a pair of $100 \mu \mathrm{m}$ pitch GSG Infinity ${ }^{\circledR}$ probes. The vector network analyzer (VNA) used for $S$-parameters measurements is an E8361A (PNA) operating up to $67 \mathrm{GHz}$ from Keysight ${ }^{\circledR}$. The intermediate frequency (IF) bandwidth is set to $100 \mathrm{~Hz}$ and the RF signal source to $-10 \mathrm{dBm}$. The frequency range is set from $100 \mathrm{MHz}$ to $50 \mathrm{GHz}$ with a frequency step of $100 \mathrm{MHz}$ (491 points). In the following, only measurements data up to $20 \mathrm{GHz}$ are considered as above $20 \mathrm{GHz}$, frequency effects dominate. An on-wafer SOLT vector calibration was applied at the probe tips using an ISS (impedance standard substrate) from Cascade Microtech®. The measured forward reflection coefficient $S_{11}$ and transmission coefficient $S_{21}$ are shown in Fig. 2 and Fig 3 respectively. In addition, the measurement of the two-port CPW empty structure $(\mathrm{gap}=20 \mu \mathrm{m})$ is also reported.

Data reported on Fig. 2 shows a reflection coefficient close to one, indicating a high impedance mismatch between the $50 \Omega$ impedance of the measurement set-up and the high impedance of the resistive devices. However, the measured magnitudes of $S_{11}$ can be used to discriminate each case. The phase-shift of $S_{11}$ shows the same signature for all the cases considered. In addition, linear frequency behaviour is observed. Consequently, the phase-shift is mainly attributed to wave propagation through the input access pad (back and forth) whereas most of the microwave signal is reflected at the open ended pad / resistive nano-film interface.

Fig. 3(a) shows a good measurement sensitivity of the magnitude of the forward transmission coefficient $S_{21}$. The responses are nearly flat in the lower frequency band indicating that the resistance can be modelled as a lumped circuit element. When the operating frequency increases, the magnitude of $S_{21}$ is affected as frequency effects appear. Same conclusions can be drawn for the measured phase-shift, i.e. the phase-shift is negligible in the lower frequency band but microwave effects induce variations in the measured phaseshifts with frequency increase. At the first order, CPW pads capacitances, capacitance coupling (between input and output CPW pads) and propagation effects (in the upper frequency limit) are mainly responsible of the phase-shift signatures.

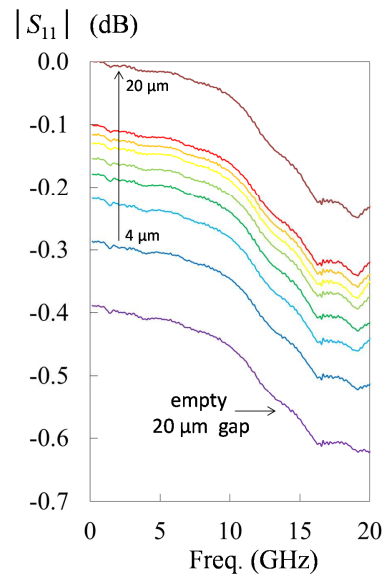

(a)

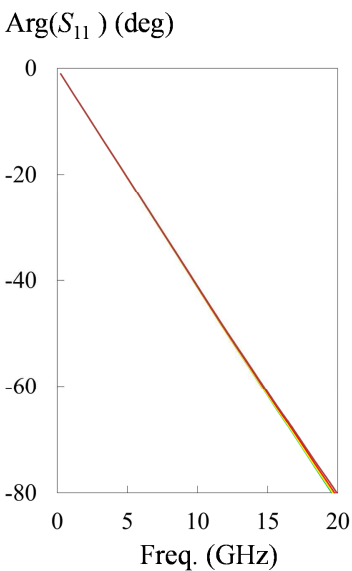

(b)
Fig. 2 Measured (a) magnitude and (b) phase-shift of the forward reflection coefficient $S_{11}$ for CPW structures with integrated nano-resistances in gaps varying from 4 to $20 \mu \mathrm{m}$.

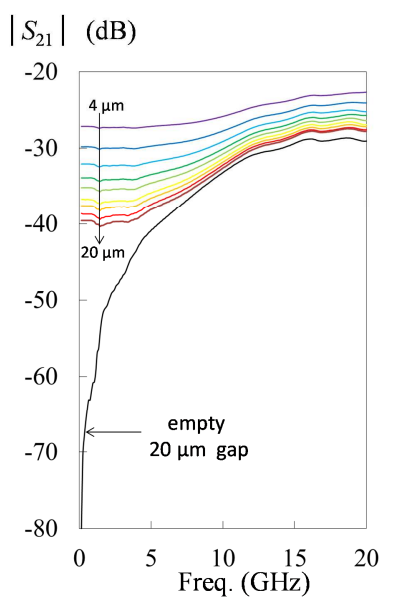

(a)

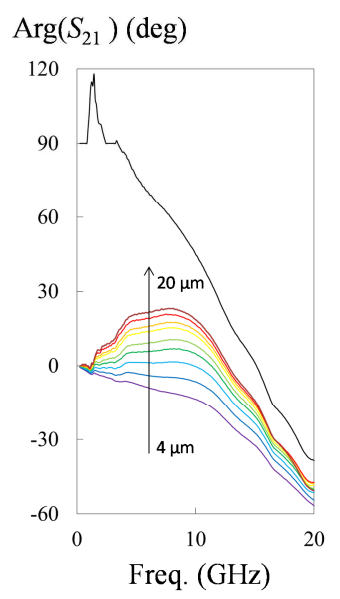

(b)
Fig. 3 Measured (a) magnitude and (b) phase-shift of the forward transmission coefficient $S_{21}$ for CPW structures with integrated nano-resistances in gaps varying from 4 to $20 \mu \mathrm{m}$.

\section{B. De-embedding procedure}

A de-embedding technique is proposed to take into account the pads and propagation effects. The method consists of subtracting from the measurement data the parasitic effects related to an empty gap CPW structure (Fig. 4). In particular, the de-embedding CPW empty structure has a gap of $20 \mu \mathrm{m}$, in accordance with the largest gap of the device under tests (DUT). Indeed, the DUTs present gaps varying from 4 to $20 \mu \mathrm{m}$. It has to be mentioned that the de-embedded complex 
impedance or admittance of the DUT gives only a relative value in respect to the de-embedding structure. In addition, the procedure assumes that the CPW access pads are identical for both the DUTs and the de-embedding structure. These two last points will be discussed in Section IV.

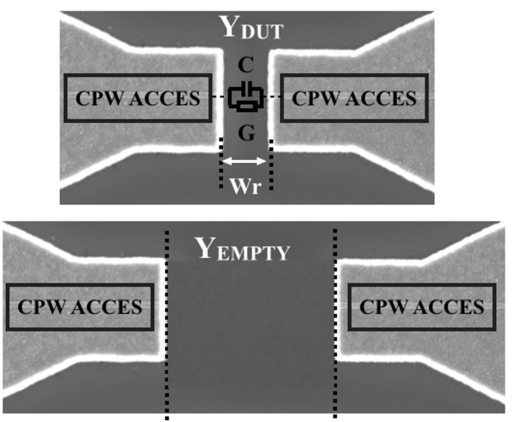

Fig. 4 SEM image and associated electrical lumped element circuit of the $4 \mu \mathrm{m}$ gap (DUT) and the $20 \mu \mathrm{m}$ gap de-embedding structure.

The measured $S$-parameters of the DUT and the de-embedding structure are converted to admittance parameters $(Y)$. The resulting admittances matrices denoted respectively as $Y_{D U T}^{M}$ and $Y_{E M P T Y}^{M}$ can be expressd as

$$
Y_{D U T}^{M}=Y_{D U T}^{I N T}+Y_{E M P T Y}^{M}
$$

Where $Y_{D U T}^{I N T}$ denotes the intrinsic admittance of the DUT. From this relative admittance matrix $Y_{D U T}^{I N T}$, both effective conductance $G=\operatorname{Real}\left(Y_{D U T}^{I N T}\right)$ and effective capacitance $C=\operatorname{Imag}\left(Y_{D U T}^{I N T}\right) / \omega$ matrices can be extracted (with $\omega$ the angular frequency). The resulting data are presented in Fig. 5 and Fig. 6.

Fig. 5 shows RF effective low conductances varying from about 0.1 to $0.5 \mathrm{mS}$ in the lower frequency regime. Flat responses are observed up to $5 \mathrm{GHz}$ whereas frequency effects noticed for the smallest gaps are attributed to the deembedding process (gap $=20 \mu \mathrm{m})$ and could be further improved by de-embedding each DUT with its corresponding CPW test structure without nano-resistance (for simplicity, only the $20 \mu \mathrm{m}$ empty gap was considered). In the same manner, Fig. 6 shows effective capacitances with flat responses from 5 to $10 \mathrm{GHz}$. Reactive effects in the low and high frequency domains that affect the measured data are linked to the de-embedding and other reactive effects (not taken into account in this $1^{\text {st }}$ order modeling). It has to be noticed that the capacitances values are determined in respect to the de-embedding $20 \mu \mathrm{m}$ gap structure and cannot be considered as absolute values. In fact, the quantitative determination of $C_{12}$ would require fine modeling of the deembedding structure. In other words, the negative effective capacitance data obtained for some of the DUTs denote relative values only. The effective capacitance values are in the order of only hundreds of aF with capacitance variations between the DUTs of tens of aF.

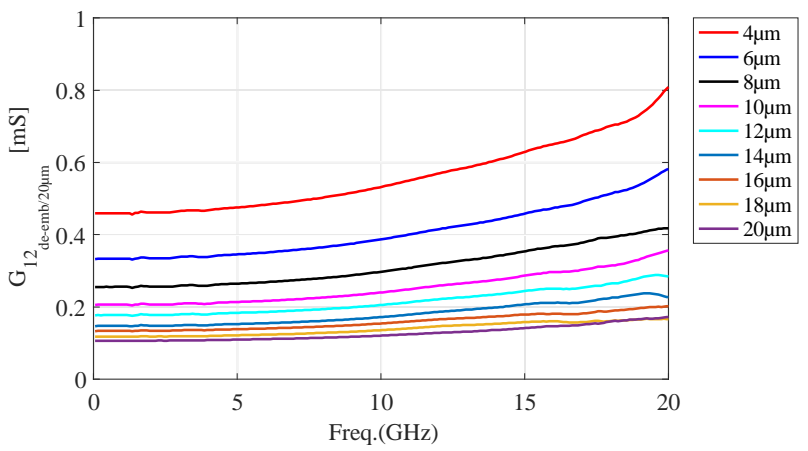

Fig. 5. Relative effective conductance versus frequency for different gap lengths. The data were de-embedded with respect to the $20 \mu \mathrm{m}$ gap

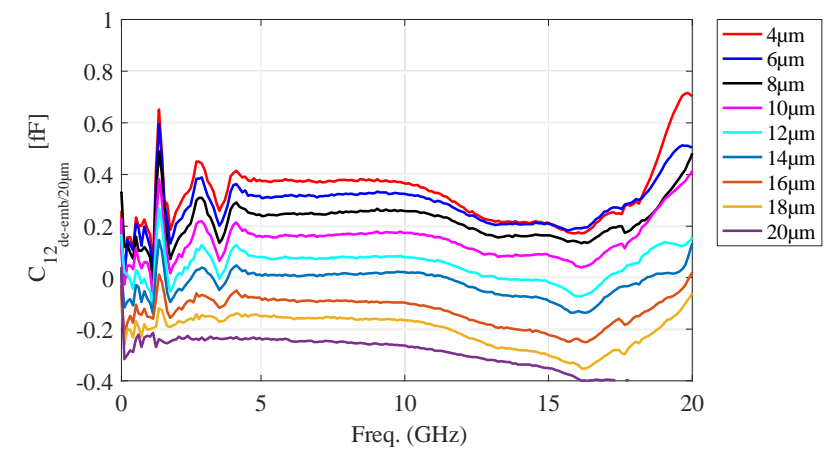

Fig. 6. Relative effective capacitance versus frequency for different gap lengths. The data were de-embedded with respect to the $20 \mu \mathrm{m}$ gap.

\section{DISCUSSION}

To resume the experiments from the previous section, thin resistive nano-films exhibit relatively low effective conductance values together with parallel effective capacitances in the order of hundreds of $\mathrm{aF}\left(10^{-18} \mathrm{~F}\right)$. The question arises regarding the measurement accuracy in the determination of such small quantities. Indeed, errors due to the hardware imperfections, i.e. the vector network analyzer, those imputable to probe contact repeatability, those related to the definition of the calibration standards and de-embedding structure and those arising from the manufacturing process seem to be not in favour of the determination of quantitative low values. In this effort, methods to lower the measurement uncertainties are first proposed. Then, the impact of (i) the post-calibration residual errors terms and (ii) the technological process variability using FEM-based EM modelling on the determination of low-value conductance and capacitance are considered.

\section{A. Lowering the set-up measurement uncertainty}

The device characterization is performed in a CPW seriesthrough measurement configuration to tackle the issue of extreme impedance microwave measurement. Indeed, the technique benefits from the measurement sensitivity and accuracy in transmission mode much better than conventional one-port measurement configuration limited by directivity errors. Moreover, stochastic errors such as drift become predominant above $20 \mathrm{GHz}$. Therefore, quantitative analysis should be ideally performed below $20 \mathrm{GHz}$ including in the ultra-low frequency regime that offers a broad range for 
electrical mechanisms studies [6]. Finally, low-case measurement uncertainty can be achieved if the full measurement campaign (including calibration, de-embedding) are performed in a short period to lower stochastic errors such as drift inherent to environmental variations.

\section{B. Uncertainty related to the vector calibration}

The method starts with the determination of the terms $\delta$, $\tau_{1}, \mu_{1}, \tau_{2}$ and $\mu_{2}$ that are the post-calibration residual calibration error terms of the calibrated VNA obtained for $n$ different vector calibrations ( $n=6$ in the present study) [7]. The complex values of the post-calibration residual error terms are extracted as the mean complex distance related to successive vector calibrations. These non-systematic residual errors that include the random (noise, connector repeatability, probe contact repeatability), the drift and stability effects between two measurements are quantified and propagated to the DUT using the following partial differential equations

$$
\Delta X_{i j} \approx \sqrt{\left(\frac{\partial X_{i j}}{\partial\left|S_{i j}\right|} \Delta \mid S_{i j}\right)^{2}+\left(\frac{\partial X_{i j}}{\partial\left|\varphi_{i j}\right|} \Delta \varphi_{i j}\right)^{2}}
$$

where $X_{\mathrm{ij}}$ denotes $G_{\mathrm{ij}}$ or $C_{\mathrm{ij}}$. The method has been applied at the test frequencies 5,10 and $20 \mathrm{GHz}$ for the DUT with $4 \mu \mathrm{m}$ gap (TABLE II).

\section{Admittance sensitivity related to the CPW critical dimensions}

The determination of the sensitivity inherent to the manufacturing process variability is studied by FEM based electromagnetic modelling using $\mathrm{CST}^{\mathrm{TM}}$ software. First, a model parameterization using nominal and measured dimensions from TABLE I is carried out to identify the critical parameters for subsequent FEM-based EM modelling of the CPW structures. Then, the uncertainties of the various device parameters are propagated through EM simulations to the DUT (TABLE II).

First, the uncertainty related to the set-up shows that capacitances values as low as hundreds of aF can be accurately measured with a capacitance uncertainty two order of magnitudes lower than the capacitance value itself. Conductance uncertainty as low as the $\mu \mathrm{S}$ is achievable for conductance value in the order of hundreds of $\mu \mathrm{S}$.

Regarding the EM analysis, differences are observed between the measured and simulated admittances as many factors influence the results (meshing, material properties, interface between substrate and resistive nano-film). Nevertheless, simulated data show same frequency evolution than their measured counterparts for all $S$-parameters. The simulation data are very instructive as they show that the variability process induces measurement error of $366 \mathrm{aF}$ and $264 \mu \mathrm{S}$ for simulated capacitance of $360 \mathrm{aF}$ and $540 \mu \mathrm{S}$ respectively at $5 \mathrm{GHz}$. Same conclusions are drawn for other test frequencies. These findings shows that hybrid characterizations (dimensions, material properties) using microscopy techniques should be ideally done prior to the microwave characterization to yield quantitative values.
TABLE II

UNCERTAINTY AND SENSITIVITY ANALYSIS ON A $4 \mu \mathrm{M}$ DEVICE

\begin{tabular}{|c|c|c|c|c|}
\cline { 2 - 5 } \multicolumn{1}{c|}{} & Frequency & $5 \mathrm{GHz}$ & $10 \mathrm{GHz}$ & $20 \mathrm{GHz}$ \\
\hline \hline Uncertainty & $C_{12 \_ \text {Meas }}(f F)$ & 0.61 & 0.63 & 1.15 \\
from $B$ & $\Delta C_{12}(a F)$ & 0.5 & 1.0 & 3.5 \\
\cline { 2 - 5 } (analytical & $G_{12 \_ \text {Meas }}(m S)$ & 0.36 & 0.41 & 0.64 \\
modeling) & $\Delta G_{12}(\mu S)$ & 0.2 & 0.4 & 1.0 \\
\hline Sensitivity & $C_{12 \_S I M}(f F)$ & 0.36 & 0.37 & 0.26 \\
from $C$ & $\Delta C_{12 \_S I M}(a F)$ & 366 & 303 & 480 \\
\cline { 2 - 5 } (EM modeling) & $G_{12 \_S I M}(m S)$ & 0.54 & 0.63 & 1.31 \\
& $\Delta G_{12 \_S I M}(\mu S)$ & 264 & 311 & 670 \\
\hline
\end{tabular}

\section{CONCLUSION}

On-wafer microwave characterization of high impedance nano-devices consisting of metallic resistive nano-films embedded in tapered CPW two-port structures have been demonstrated up to $20 \mathrm{GHz}$. From the experiments, accurate extraction of microwave effective conductance and capacitance in the order of the $\mu \mathrm{S}$ and lower than the $\mathrm{fF}$ are demonstrated respectively. In addition, uncertainty study related to the vector calibration together with a study related to the admittance sensitivity were proposed to support the experimental data. These results altogether are instructive for the establishment of planar microwave measurements of nanodevices and form the basis for future traceability of microwave measurements at the nanoscale.

\section{ACKNOWLEDGMENT}

This work is performed under EMPIR Planarcal projects (http://www.planarcal.ptb.de). This work used the facilities within the EQPX ExCELSiOR project.

\section{REFERENCES}

[1] H. Happy, K. Haddadi, D. Théron, T. Lasri, and G. Dambrine, "Measurement techniques for RF nanoelectronic devices : new equipment to overcome the problems of impedance and scale mismatch," IEEE Microwave Magazine, vol. 15, no. 1, pp. 30-39. Jan. 2014.

[2] Z. Yu and P. J. Burke, "Microwave transport in single-walled carbon nanotubes" Nano Letters, vol. 5, no. 7, pp. 1403-1406, July 2005.

[3] T. Wallis, A. Imtiaz, H. Nembach, K. A. Bertness, N. A. Sanford, P. T. Blanchard and P. Kabos., "Calibrated broadband electrical characterization of nanowires," 2008 Conference on Precision Electromagnetic Measurements Digest, Broomfield, pp. 684-685, CO, 2008.

[4] U. Arz, S. Zinal, T. Probst, G. Hechtfischer, F. J. Schmückle and W. Heinrich, "Establishing traceability for on-wafer S-parameter measurements of membrane technology devices up to $110 \mathrm{GHz}, " 2017$ 90th ARFTG Microwave Measurement Symposium (ARFTG), Boulder, CO, USA, 2017, pp. 1-4.

[5] L. Galatro and M. Spirito, "Millimeter-Wave On-Wafer TRL Calibration Employing 3-D EM Simulation-Based Characteristic Impedance Extraction," IEEE Transactions on Microwave Theory and Techniques, vol. 65, no. 4, pp. 1315-1323, Apr. 2017.

[6] K. Daffé, G. Dambrine, F. von Kleist-Retzow, and K. Haddadi, "RF wafer probing with improved contact repeatability using nanometer positioning," in 2016 87th ARFTG Microwave Measurement Conference (ARFTG), 2016, pp. 1-4

[7] D. K. Rytting, "Network analyzer accuracy overview », in $58^{\text {th }} A R F T G$ Conference Digest, 2001, vol. 40, pp. 1-13. 